\title{
Type 1 Kounis syndrome presenting as acute coronary syndrome in the context of red dye-induced anaphylaxis
}

\author{
David M Kepecs ${ }^{1}$ (1) $\cdot$ Jimmy J Zhang $^{1} \cdot$ Habibat A Garuba ${ }^{1} \cdot$ Angeline Law $^{1}$
}

Received: 2 February 2021 / Accepted: 24 July 2021 / Published online: 10 August 2021

() The Author(s), under exclusive licence to Canadian Association of Emergency Physicians (CAEP)/ Association Canadienne de Médecine d'Urgence (ACMU) 2021

Keywords Anaphylaxis · Acute coronary syndrome · Emergency medicine

\section{Introduction}

Kounis syndrome, otherwise known as allergic angina, is defined as acute coronary syndrome following an allergic insult. While Kounis syndrome is a documented disease entity, it is not well understood among clinicians and is often overlooked. Here, we present the case of type I Kounis syndrome in a 31-year-old male who presented with non-ST elevation myocardial infarction following an anaphylactic episode caused by ingesting tetrahydrocannabinol (THC) edibles containing artificial red dye.

\section{Case presentation}

A 31-year-old Indigenous-Canadian male $(\mathrm{BMI}=52.3 \mathrm{~kg} /$ $\mathrm{m}^{2}$ ) presented to the emergency department (ED) with a 2-h history of chest pain. He had no significant medical history aside from multiple anaphylactic episodes caused by artificial red dyes. The patient described an anaphylactic reaction to red dye containing THC edibles he consumed. His symptoms included hives, angioedema and shortness of breath. He had no presyncope or gastrointestinal symptoms. He had not used an EpiPen. His anaphylactic symptoms resolved after $2 \mathrm{~h}$ with antihistamine treatment, but he developed chest pain prompting his presentation to the ED.

On arrival, the patient endorsed $9 / 10$ chest pain at rest. His initial vitals and laboratory results are contained in Table 1. An electrocardiogram (ECG) showed sinus

David M Kepecs

dkepecs@toh.ca

1 The Ottawa Hospital, 501 Smyth Road, Ottawa, ON K1H 8L6, Canada tachycardia with new T wave inversions in lead III (Supplementary figure S1). Serial ECGs did not show ST elevations or depressions.

Initial cardiac point of care ultrasound performed in the ED by a cardiologist demonstrated global systolic dysfunction of the left ventricle (LV). The LV was dilated with a reduced left ventricular ejection fraction (LVEF) of 40-45\%. The right ventricle was of normal size and function. There were no signs of valvular disease or pericardial effusion. Formal echocardiogram on day 1 of admission showed a dilated $\mathrm{LV}$ with recovered ejection fraction $(\mathrm{LVEF}=50-55 \%)$. The initial deterioration in LV function was likely attributed to myocardial stunning following the onset of Kounis syndrome. However, we cannot exclude operator variability, since echocardiograms were performed and interpreted by different providers.

The patient received ASA (160 mg orally), Ticagrelor (180 mg orally) and IV heparin infusion. Chest pain resolved after $12 \mathrm{~h}$. Considering the above findings and his elevated troponins, cardiac catheterization was performed on Day 5. Catheterization revealed no obstructive coronary artery disease. The patient was discharged with allergy/immunology follow-up.

\section{Discussion}

The pathophysiology of Kounis syndrome involves the interaction between mast cells and other macrophages and $\mathrm{T}$ cells. When mast cells degranulate, histamine and several inflammatory mediators (e.g., tryptase, thromboxane and leukotrienes) are released. It is hypothesized that each of these inflammatory mediators, with macrophage and T-cell activation, stimulate platelet aggregation and degradation of the collagen cap inducing plaque rupture [4]. In addition, 
Table 1 Triage vitals and laboratory values

\begin{tabular}{llll}
\hline Triage vitals & & Relevant bloodwork & \\
\hline Temperature & $37 .{ }^{\circ} \mathrm{C}$ & Hemoglobin & $151 \mathrm{~g} / \mathrm{L}$ \\
Blood pressure & $112 / 93 \mathrm{mmHg}$ & White blood cell count & $24.1 \times 10^{9} / \mathrm{L}$ \\
Heart rate & 110 beats/minute & Platelets & $497 \times 10^{9} / \mathrm{L}$ \\
Respiratory rate & 19 breaths/minute & HbA1c & $5.8 \%$ \\
Oxygen saturation & $97 \%$ & LDL & $1.3 \mathrm{mmol} / \mathrm{L}$ \\
& & High-sensitivity troponin (initial) & $45 \mathrm{ng} / \mathrm{L}$ \\
& & High-sensitivity troponin peak (Day 1$)$ & $508 \mathrm{ng} / \mathrm{L}$ \\
\hline
\end{tabular}

histamine increases vascular permeability and mediates coronary artery vasoconstriction, which contributes to coronary vasospasm.

Here, we present the first reported case of red dyeinduced Kounis syndrome. On review of ECGs performed, there were no electrocardiographic signs suggestive of cardiac ischemia. This may be explained by our patient's delayed presentation to the ED following anaphylaxis. In addition, Abdelghany et al. presented a case series of Kounis syndrome that involved patients with mostly abnormal ST segments. Only 3 of 175 patients had normal ECGs [1]; this patient, with non-specific changes, possibly fits into this minority.

The differential diagnosis included pericarditis, acute coronary syndrome and myocarditis. The patient did not meet any diagnostic criteria for pericarditis. Coronary angiogram revealed normal coronary vasculature, decreasing the probability of type I acute coronary syndrome. Myocardial Infarction with Nonobstructive Coronary Arteries (MINOCA), including myocarditis and type I Kounis syndrome, was considered. While cardiac MRI is the most accurate diagnostic tool for myocarditis, it could not be performed because of the quick recovery of the patient, social demands pressuring the patient's return home, and resource constraints, since cardiac MRI required transfer to a nearby center. The correlation between the patient's allergic reaction, chest pain and quick recovery strongly indicate type I Kounis syndrome.

Kounis syndrome was first described in 1991 by Kounis and Zafras as a mast cell degranulation-mediated coronary artery vasospasm [2]. There are 3 proposed variants of Kounis syndrome. Type I (72.6\% of cases) involves either coronary vasospasm with normal coronary arteries that do not have raised cardiac markers, or coronary vasospasm that progresses to myocardial infarction and raised cardiac markers. Type II (22.3\% of cases) involves pre-existing atherosclerotic disease with the allergic reaction inducing plaque rupture presenting as acute coronary syndrome. Type III (5.1\% of cases) involves coronary stent thrombosis [3, 4].

Type I and type II Kounis syndrome present similarly, especially when cardiac markers are elevated. Clinicians should treat the underlying anaphylaxis and acute coronary syndrome in both subgroups. Exceptionally, where patients with type I Kounis syndrome demonstrate coronary vasospasm without laboratory evidence of myocardial ischemia, clinicians should simply treat the underlying allergic response. Patients with type III Kounis syndrome should be managed like patients with type I and II, but they also require intra-stent aspiration.

Clinicians should always treat the underlying allergic response in Kounis syndrome. The only life-saving intervention in anaphylaxis is epinephrine; however, we caution that epinephrine can aggravate myocardial ischemia and induce coronary vasospasm. Supportive care involves antihistamine treatment which reduce allergic symptoms, and halts the histamine response that leads to coronary vasospasm or plaque rupture. Opioids should be used with caution for pain management. They can cause mast-cell degranulation and may aggravate the allergic response.

In patients treated for acute coronary syndrome, clinicians should start dual anti-platelet therapy and anticoagulation, and consider vasodilator drugs. Nitroglycerin, a potent dilator of coronary vessels, is the most effective drug for treating hypersensitivity-induced coronary vasospasm that characterizes Kounis syndrome. However, we caution this treatment since nitroglycerin may worsen hypotension in the context of anaphylaxis.

The European Society of Cardiology guidelines recommend early initiation of beta-blockers in patients with ongoing ischemic symptoms without contraindications [5]. Beta-blockers are contraindicated in Kounis syndrome [6]. Beta blockade may be problematic in biphasic allergic reactions by reducing the efficacy of epinephrine, which is needed to increase cardiac 
output and bronchodilation. Glucagon should be considered to reverse beta blockade in patients on beta blocker therapy [7].

\section{Conclusion}

Physicians should consider Kounis syndrome in patients who present with acute coronary syndrome and allergy symptoms or a history of anaphylaxis. Future research should delineate the differences in management and treatment therapies of Kounis syndrome and type 1 acute coronary syndrome.

Supplementary Information The online version contains supplementary material available at https://doi.org/10.1007/s43678-021-00186-9.

\section{Declarations}

Conflict of interest No competing financial interests exist. There are no potential conflicts of interest.

\section{References}

1. Kounis NG. Kounis syndrome (allergic angina and allergic myocardial infarction): a natural paradigm? Int J Cardiol. 2006;110(1):7-14.
2. Kounis NG, Zavras GM. Histamine-induced coronary artery spasm: the concept of allergic angina. Br J Clin Pract. 1991;45(2): 121.

3. Sciatti E, Vizzardi E, Cani DS, Castiello A, Bonadei I, Savoldi D, et al. Kounis Syndrome a disease to know: case report and review of the literature. Monaldi Arch Chest Dis. 2018;88(1):898.

4. Kounis NG. Coronary hypersensitivity disorder: the Kounis syndrome. Clin Ther. 2013;35(5):563-71.

5. Collet JP, Thiele H, Barbatoa E, et al. 2020 ESC Guidelines for the management of acute coronary syndromes in patients presenting without persistent ST-segment elevation: The Task Force for the management of acute coronary syndromes in patients presenting without persistent ST-segment elevation of the European Society of Cardiology (ESC). Eur Heart J. 2021;42(14):1289-367.

6. Abdelghany M, Subedi R, Shah S, Kozman H. Kounis syndrome: a review article on epidemiology, diagnostic findings, management and complications of allergic acute coronary syndrome. Int J Cardiol. 2017;1(232):1-4.

7. Shaker MS, Wallace DV, Golden DB, Oppenheimer J, Bernstein JA, Campbell RL, et al. Anaphylaxis-a 2020 practice parameter update, systematic review, and Grading of Recommendations, Assessment, Development and Evaluation (GRADE) analysis. J Allergy Clin Immunol. 2020;145(4):1082-123. 